\title{
Analysis of polymer grafted inside the porous hydrogel using confocal laser scanning microscopy
}

\author{
I. N. Savina ${ }^{1,2}$, M. Tuncel $^{3}$, A. Tuncel ${ }^{4}$, I. Yu. Galaev ${ }^{*}$, B. Mattiasson ${ }^{1}$ \\ ${ }^{1}$ Department of Biotechnology, Center for Chemistry and Chemical Engineering, Lund University, Box 124, SE-22100, \\ Lund, Sweden \\ ${ }^{2}$ Protista Biotechnology AB, P.O. Box 86, SE-26722 Lund, Sweden \\ ${ }^{3}$ Department of Anatomy, Faculty of Medicine, Hacettepe University, 06532 Beytepe, Ankara, Turkey \\ ${ }^{4}$ Chemical Engineering Department, Hacettepe University, 06532 Beytepe, Ankara, Turkey
}

Received 29 January 2007; accepted in revised form 24 March 2007

\begin{abstract}
Graft polymerization of glycidyl methacrylate onto the pore surface of polyacrylamide macroporous gel was implemented in DMSO-aqueous solution using diperiodatocuprate(III) complexes as an initiator. The grafting densities up to $410 \%$ were achieved. The graft polymerization was confirmed by gravimetrical methods and FTIR. The graft polymerization of polymer inside the pores of the macroporous gel resulted in increased flow resistance through the gel matrix. The distribution of grafted polymer on the gel pore surface material was studied by scanning electron microscopy (SEM) and confocal laser scanning microscopy (CLSM). CLSM is an alternative method for studying morphology of gel surface with grafted polymer having the advantages over the SEM allowing to investigate the distribution of grafted polymer inside the hydrogel in a native hydrated state. The microscopic techniques demonstrated uneven distribution of the grafted polymer inside the gel pores as a result of initiating the graft polymerization by insoluble initiator deposited on the pore surface.
\end{abstract}

Keywords: polymer gels, material testing, graft polymerization, confocal laser scanning microscopy

\section{Introduction}

Hydrogels with mobile polymer chains terminally or loop-wise attached to the hydrogel polymer backbone are of interest for different applications in the biotechnological, medical and pharmaceutical field. Generically, polymer chains of various nature (hydrophilic-hydrophobic, ion-exchange, stimuli-responsive polymer chains) could be introduced into the hydrogel backbone by graft polymerization providing hydrogels with additional behaviour. Thus the wettability, biocompatibility, and water content of the hydrogels were improved by grafting polymer chains from polymer backbone [1]. The terminally attached polymer chains are flexible and capable of fast changing their conformation. This feature has been used for increasing the swelling degree and swelling rate of hydrogels [2] and to accelerate the rate of shrinking/swelling of stimuli-sensitive hydogels in response to changes of external condition ( $\mathrm{pH}$, temperature) $[3$, 4]. The localization of functional groups (cationexchange, anion-exchange, affinity, etc.) at the polymer chains terminally attached to hydrogel surface makes it possible to increase the loading capacity and mass transfer rates and results in marked changes in selectivity of hydrogels during their interactions with biological nano- and macroparticles $[5,6]$. Thus the grafted hydrogels have attracted a considerable attention as promising materials for producing new stimuli-responsive drug delivery systems [7-9], biomimetic actuators [10] or chromatographic materials [6]. 
The hydrogels with terminally bound polymer chains could be prepared by different methods. Hydrogels with freely mobile polymer chains terminally attached onto polymer backbone were prepared by co-polymerization of monomer mixture containing macromonomer $[4,5,11]$ or as a result of cross-linking of preformed grafted polymer [12]. Grafting polymers onto an already prepared gel surface is another method, commonly used for gel surface modification. This could be implemented using grafting to method, when the preformed polymer chains with terminally functional groups covalently bound to the surface [13], or using grafting from method, when the polymer chains are grown via monomer polymerization initiated from the active sites on the gel surface [14]. When comparing these two methods, the grafting from method allows the formation of grafted layer with higher density and thickness of polymer brushes, which could be controlled by the amounts of monomer and catalyst used. The formation of high density of polymer brushes using grafting to method is difficult because of steric crowding of reactive sites at the gel surface by already bound polymer chains.

The structure of grafted layer, thickness, density and distribution of polymer grafted in the bulk of hydrogel, is very important considering controlling the gel behavior. Introduction of additional grafted polymer into the gel backbone is usually characterized by using gravimetric methods, infrared spectral analysis and different scanning calorimetry. The determination of the structure of polymer layer grafted on the surface of hydrogel presents especially a big challenge. In this case, the polymer brushes could not be analyzed by techniques traditionally used for the analysis of polymer brushes grafted on flat surfaces like AFM, ellipsometry, surface plasmon resonance or using quartz crystal microbalance. The microscopic methods give the possibility to look inside the bulk structure of polymer materials and visualize the surface morphology of the porous polymer material. Scanning electron microscopy (SEM) has been used for the study of the surface morphology of grafted hydrogels [1]. It reveals the formation of additional polymer layer on the surface of hydrogels. However, sample preparation for SEM requires drying the sample followed by metal coating that might alter hydrogel morphology and the picture obtained represents grafted polymer layer in dehydrated state. This problem could be solved by applying confocal laser scanning microscopy (CLSM). CLSM offers advantages over conventional SEM procedures as it does not require special water removal prior to examination that allows for studying the bulk structure of hydrogel samples in their native state [15-17].

CLSM is a technique to gain high resolution views of thick specimens by rejection of out of focus scattering. Through the use of a pinhole aperture, light emerging from material above and below the plane of interest is effectively blocked, resulting in the detection of light from a thin specimen slide that is centred on the focal plane. This feature of CLSM, known as 'optical sectioning', makes it possible to scan at various $x-y$ planes corresponding to different depths of the sample, and, thus to reconstruct the 3-D region of the specimen. CLSM has been widely used for investigation of the bulk structure of polymer materials [15-17]. However to the best of our knowledge there were no reports on the study of polymer grafted on the surfaces in the bulk of porous hydrogel material. Studies on grafted polymers with CLSM are mainly restricted to flat surfaces. For example, Nakayama et al. studied the thickness of grafted polymer layer on the surface of PET films using CLSM [18]. The grafted surface was treated by staining with rose bengal, which fluorescence light was observed under a fluorescent microscope. The fluorescence intensity, which can be correlated to the graft yields, provides the information about thickness of grafted polymer layer on the polymer film.

In this study we have attempted to use CLSM for studying the distribution of grafted polymer on the pore surface in the bulk of porous gel material. Glycidyl methacrylate (GMA) was grafted onto the surface of macroporous polyacrylamide gel, so called cryogel (pAAm cryogel) [19], using diperiodatocuprate(III) as initiator. The gel pore surface morphology and distribution of grafted polymer in the bulk of grafted pAAm cryogel were studied by SEM and CLSM.

\section{Experimental part}

\subsection{Materials}

Glycidyl methacrylate (GMA, $\geq 97 \%$ purity) was from Fluka (Seelz, Germany) and used without additional purification. Acrylamide (AAm, more 
than $99.9 \%$ purity, electrophoresis reagent), methylene-bis-acrylamide (MBAA, 99\%), N,N,N',N'tetra-methyl-ethylenediamine (TEMED, 99\%), ammonium persulfate (APS, 98\%) and fluoresceinamine, isomer I were from Aldrich (Steinheim, FRG). The potassium diperiodatocuprate(III) solution contained $0.056 \mathrm{M}$ of $\mathrm{Cu}$ (III) was prepared according to the method described previously [20]. The rest of chemicals were of the best quality available.

\subsection{Methods}

\subsubsection{Preparation of pAAm cryogels}

Dry pAAm cryogels were provided by Protista Biotechnology AB (Lund, Sweden). The pAAm cryogels were prepared in glass tubes from polymerization medium containing $6 \mathrm{w} / \mathrm{v} \%$ (AAm+ MBAA) and 8/1 AAm/MBAA, the amount of APS as well as TEMED was $1.2 \mathrm{w} / \mathrm{w} \%$ of the total monomer weight. The polymerization solution in the tubes was frozen at $-12^{\circ} \mathrm{C}$ and kept at this temperature for $20 \mathrm{~h}$. After thawing and washing with water $(200 \mathrm{ml})$ the gel matrix was dried at $60^{\circ} \mathrm{C}$ and stored in dry state.

\subsubsection{Preparation of the grafted cryogels}

Graft polymerization was carried out according to method described earlier [21]. A dried pAAm cryogel $(0.10 \pm 0.03 \mathrm{~g})$ was placed in a glass tube and saturated with initiator solution $(0.35 \mathrm{M} \mathrm{Cu}(\mathrm{III})$, $1 \mathrm{M} \mathrm{NaCl}$ ). The columns saturated with initiator solution were incubated for $10 \mathrm{~min}$ at room temperature and then washed with degassed solution of $0.5 \mathrm{M} \mathrm{NaCl}$ until $\mathrm{pH}$ of washing solution was 7.0. The samples were incubated at $40^{\circ} \mathrm{C}$ for $30 \mathrm{~min}$. The monomer solutions in DMSO $(70 \mathrm{v} / \mathrm{v} \%)$ was passed through the gel matrix at a flow rate of $2 \mathrm{ml} / \mathrm{min}$. The flow was stopped by corking the glass tube. The glass tubes were placed in waterbath at $80^{\circ} \mathrm{C}$ for $4 \mathrm{~h}$. Any soluble homopolymer of GMA was extracted from the grafted cryogels with DMSO. The un-reacted diperiodatocuprate(III) and reaction byproducts were removed by washing with $30 \mathrm{ml} 0.1 \mathrm{M} \mathrm{HCl}$. Finally, the gel was washed with an excess of deionized water.

\subsubsection{Characterization of graft cryogels}

The grafting degree $(G)$ and density $(D)$ of the graft polymerization were calculated as Equations (1), (2):

$$
\begin{aligned}
& G[\%]=\frac{W_{1}-W_{0}}{W_{0}} \cdot 100 \\
& D[\mathrm{mmole} / \mathrm{ml}]=\frac{W_{1}-W_{0}}{V} \cdot \frac{1000}{M_{w}}
\end{aligned}
$$

where $W_{0}$ and $W_{1}$, are the weights [g] of original and grafted samples, respectively. $V$ is the volume [ml] of sample, $M_{w}$ is the molecular weight of monomer used for the graft polymerization.

Flow rate of water passing through the column was measured at the constant hydrostatic pressure equal to $100 \mathrm{~cm}$ of water-column corresponding to a pressure of circa $0.01 \mathrm{MPa}$ according to Plieva et al. [22].

The FTIR spectra of dried plain cryogel and grafted cryogel in potassium bromide pellets were recorded using an FTIR-8300 spectrophotometer (Shimadzu). Scanning electron microscopy was carried out using JEOL JSM-5600LV scanning electron microscope [22].

Confocal microscopy was carried out as follows. A disc of approximately $1 \mathrm{~mm}$ in height was cut from the wet cryogel monolith. The disk was labelled with fluoresceinamine and examined in confocal laser scanning microscope (CLSM, Leica SP2, Heidelberg, Germany) equipped with $\mathrm{He}-\mathrm{Ne}$ laser. The disc was placed on a glass slide with a cover slip. CLSM images were taken from the disc interior with both maximum projection and transparency modes. The excitation and emission wavelengths were 488 and $519 \mathrm{~nm}$, respectively. All images were generated by optical sectioning in z-direction. For this purpose, 500 optical sections were taken along with a z-distance of $160 \mu \mathrm{m}$. The samples were stained with fluoresceinamine which was covalently bound to the grafted poly-GMA as follows. Dried cryogels were placed in glass tube and saturated with solution of fluoresceinamine $(50 \mathrm{mg} / \mathrm{ml}$ in aqueous solution of DMSO, $50 \mathrm{v} / \mathrm{v} \%$, $\mathrm{pH}$ 7.0). The glass tubes were sealed and placed in thermostated bath at $70^{\circ} \mathrm{C}$ for $5 \mathrm{~h}$. Then the cryogel was washed with excess of water to remove unbound fluoresceinamine.

\section{Results and discussion}

Glycidyl methacrylate (GMA) was grafted onto the pore walls of macroporous polyacrylamide hydrogel, so called cryogel (pAAm cryogel), using diperiodatocuprate as initiator. Cryogels are pro- 
duced by radical copolymerization of acrylamide with methylene-bis-acrylamide in partially frozen reaction media $[22,23]$. They are characterized by dense non-porous polymer walls and a system of large interconnected pores filled with water. The swollen cryogel fill up the glass tubes where it was prepared so that the liquid when applied on top of the gel plug passes through the interconnected porous system of the cryogel. The cryogel with mean pore size about $35 \mu \mathrm{m}$ with pore size distribution of $5-100 \mu \mathrm{m}$ was used in this study [24, 25]. The structure of large interconnected pores of cryogels promote grafting providing an ample surface for grafting and ensuring a good mass transport of reagents in the bulk of the gel matrix. Soluble (nongrafted) homopolymer and insoluble by-products of initiator oxidation formed during the polymerization reaction are easily washed out after finishing the graft polymerization.

Graft polymerization of GMA was initiated by diperiodatocuprate(III) which is an efficient initiator of radical polymerization of vinyl monomers on the polyacrylamide backbone [21, 26, 27]. Diperiodatocuprate(III) forms, via redox reaction with amide groups on the polymer backbone, amidil radicals which initiate radical polymerization (for mechanism see Zhang et al. [28]). The graft polymerization was implemented using a two-steps method: saturating the cryogels with aqueous solution of initiator and replacing it with monomer solution in aqueous-organic mixture. The initiator was deposited onto the pore surface in the form of insoluble sodium diperiodatocuprate(III) during the saturation with initiator solution. The soluble initiator and $\mathrm{KOH}$ presented in the initiator solution were removed by washing with $\mathrm{NaCl}$ (see methods). This approach allows implementing effective graft polymerization of water insoluble GMA in the DMSO-water medium and allows avoiding the high-alkaline conditions during the graft polymerization, decreasing the risk of hydrolysis of epoxy groups and the polyacrylamide backbone during the graft polymerization [21].

The cryogels with high density of grafted GMA up to $1.44 \mathrm{mmol} / \mathrm{ml}$ of cryogel (grafting percentage, $G \%=410 \%$ ) were prepared using this method. The grafting percentage $(G \%)$ and the density of GMA grafted $\left(D_{G M A}, \mathrm{mmol} / \mathrm{ml}\right.$ of cryogel) increase with increase in the monomer concentration (Table 1).

The grafting of GMA was confirmed by comparing FTIR spectra of grafted and non-grafted, plain
Table 1. The grafting percentage $(G \%)$ and the density ( $\mathrm{D}_{\mathrm{GMA}}$ ) of GMA grafted pAAm cryogel

\begin{tabular}{|c|c|c|}
\hline $\begin{array}{c}\text { Monomer concentration } \\
{[\mathbf{M}]}\end{array}$ & $\begin{array}{c}\mathbf{G} \\
{[\%]}\end{array}$ & $\begin{array}{c}\mathbf{D}_{\text {GMA }} \\
{[\mathbf{m m o l} / \mathbf{m l} \text { of cryogel }]}\end{array}$ \\
\hline 0.1 & 15 & 0.05 \\
\hline 0.3 & 79 & 0.28 \\
\hline 0.5 & 150 & 0.53 \\
\hline 0.7 & 191 & 0.67 \\
\hline 1.0 & 275 & 1.00 \\
\hline 1.5 & 410 & 1.44 \\
\hline
\end{tabular}

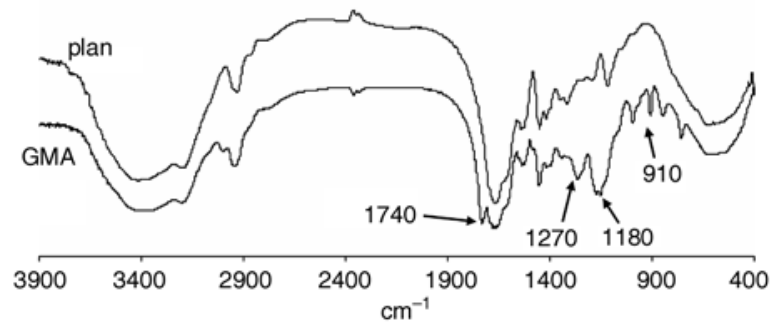

Figure 1. FTIR-spectra of non-grafted (plain) and GMAgrafted pAAm cryogels

cryogel. In FTIR spectrum of GMA-grafted pAAm cryogel, the strong band of $\mathrm{C}(=\mathrm{O})-\mathrm{O}$ stretching vibration appeared in the region $1270 \mathrm{~cm}^{-1}$ (Figure 1). This band together with the $\mathrm{vC}=\mathrm{O}$ at $1740 \mathrm{~cm}^{-1}$ revealed the ether structure in the grafted polymer [29]. The presence of the $910 \mathrm{~cm}^{-1}$ band indicated the incorporation of epoxy groups [29]. There is the strong band at $1170 \mathrm{~cm}^{-1}$ in the spectrum of GMA-grafted pAAm cryogel. This band could be related to $\mathrm{C}-\mathrm{N}$ stretching vibration and $\mathrm{NH}$ in-plane deformation of secondary amides [29]. These data confirm initiation of graft polymerization from the amide group of redox reaction of diperiodatocuprate(III) with amide groups on the polymer backbone pAAm cryogel $\left(-\mathrm{C}(=\mathrm{O}) \mathrm{NH}_{2}\right)$ and formation of grafted polymer chain $(-\mathrm{C}(=\mathrm{O}) \mathrm{NH}-$ polyGMA $)$.

The graft polymerization of the polymer inside the cryogel resulted in increased flow resistance of the cryogel (Figure 2). Previously, it was demonstrated that measuring the flow rates through the grafted pAAm cryogel monoliths at the constant pressure of water column represents a simple method for the comparative study of pore structure of the cryogel samples and the changes in the pore structure imposed additionally by the grafted polymer layer [30]. The presence of the high concentration of the grafted polymer inside the pores localized near the pore surface obstructed the flow of solutes through the GMA-grafted pAAm cryogel matrix increasing the flow resistance. 


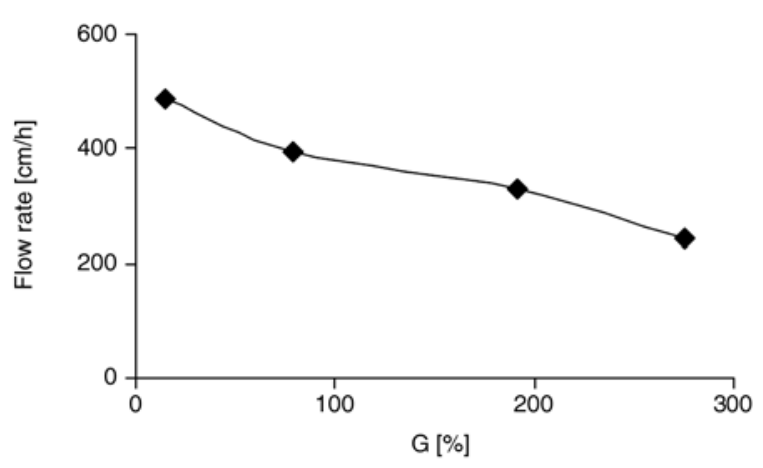

Figure 2. Flow rate of water through the GMA-grafted cryogel

The distribution of the grafted polymer within the pore of the cryogel was studied by SEM. SEM clearly demonstrated the formation of newlyformed polymer phase inside the cryogel pores (Figure 3, for structure of non-grafted pAAm cryogel see Figure 2 in Plieva et al. [23]). The grafted polymer is visible in the form of particles of different shape and size, which are unevenly distributed over the polymer surface of the cryogel walls (Figure 3). There are areas with small particles (less then $1 \mu \mathrm{m})$ 'evenly' distributed within restricted area together with the particles of large size up to $20 \mu \mathrm{m}$ (Figure 3a, b). Moreover the rod- and star- shaped particles of the grafted polymer of the size ca. $100 \mu \mathrm{m}$ are also present (Figure $3 \mathrm{c}, \mathrm{d}$ ). As the graft polymerization has been initiated with insoluble initiator, sodium diperiodatocuprate(III) complexes, deposited at the surface of the pores, the uneven distribution of the insoluble initiator could result from the efficient synthesis of grafted polymer occurring at specific sites rather than producing molecular brushes covering evenly the surface of pores. Thus the SEM demonstrated that the graft polymerization initiated by initiator deposited at the surface resulted in an uneven distribution of grafted polymer and formation of the small and large polymer pieces of grafted polymer in the cryogel pores.

However, when using SEM the cryogel sample must be dried before investigation thus it could represent the altered structure of the grafted cryogel. Therefore, in our study we have attempted to use CLSM for the characterization of the bulk structure of the grafted cryogel. With this technique water does not need to be removed prior to examination and the surface morphology of the cryogel and the layer of grafted polymer could be studied in hydrated state and in much depth compared to
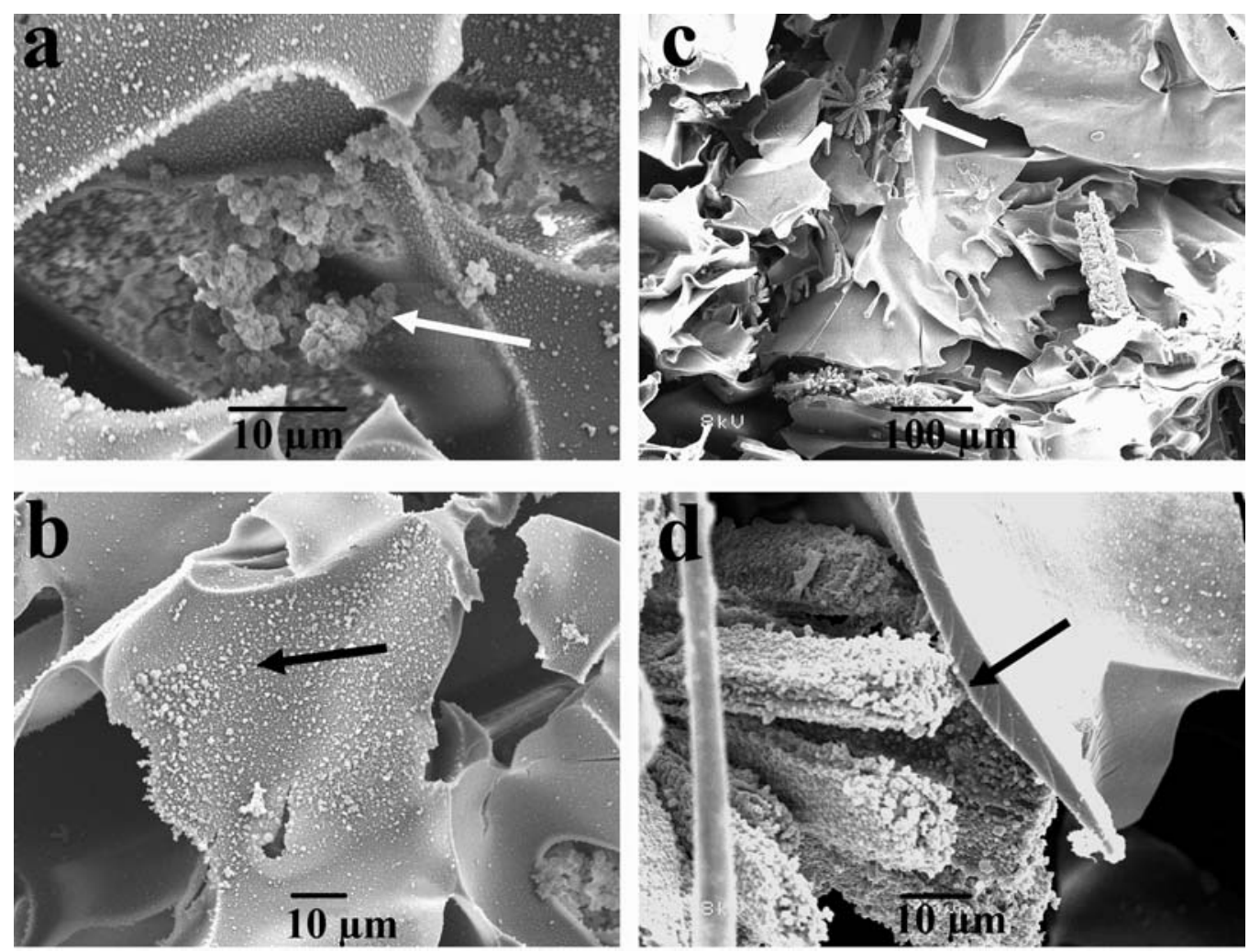

Figure 3. SEM microphotograph of diametrical cross-sections of GMA-grafted cryogel (G\% 130\%). The grafted polymer is visible in the form of small particles 1-20 $\mu \mathrm{m}$ size (a and b), 'evenly' distributed within restricted area (b) and formed aggregates (a). The rod- and stars-shaped particles of large size ca. $100 \mu \mathrm{m}$ also presented (c and d) 

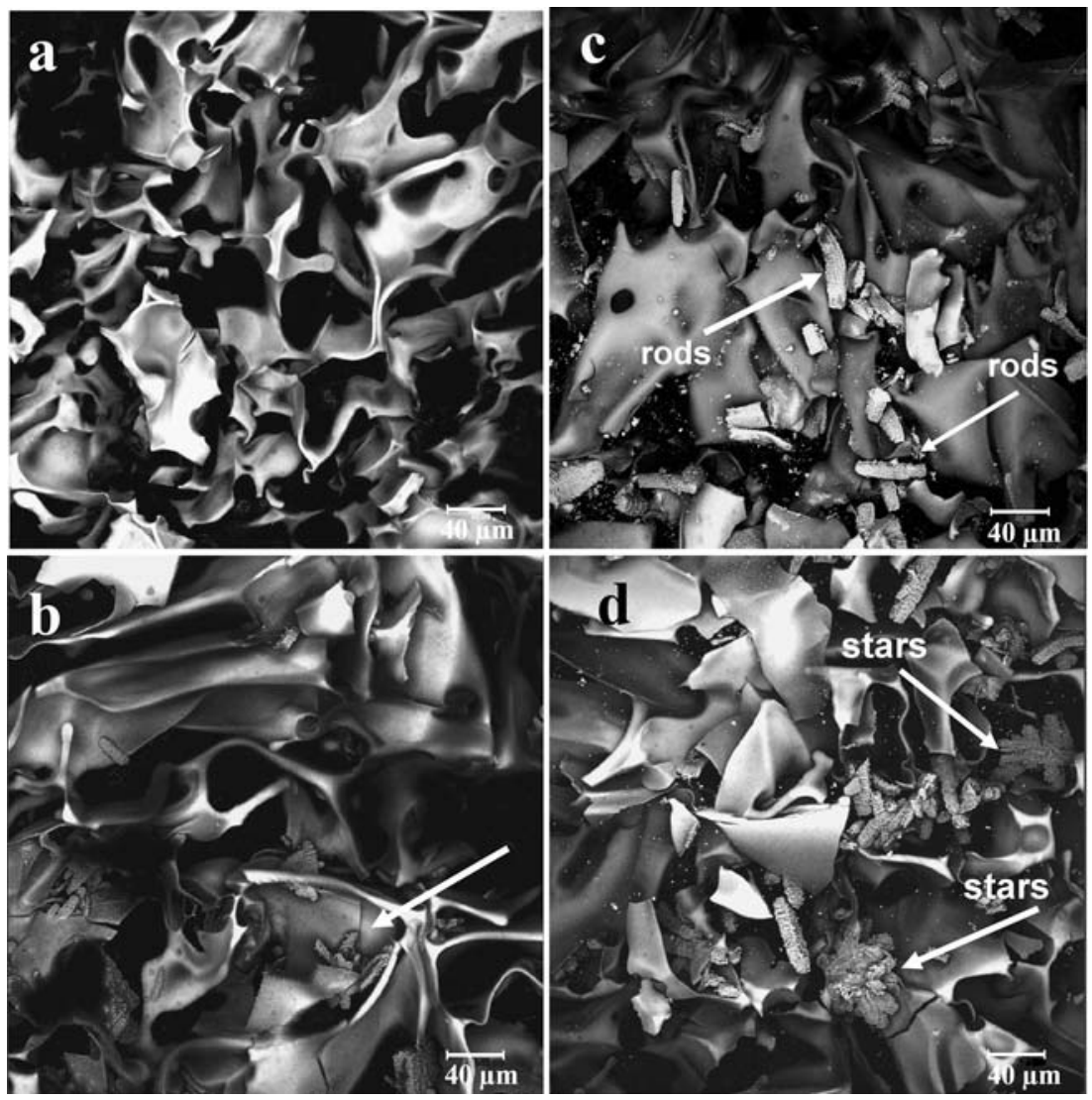

Figure 4. CLSM microphotographs of non-grafted (a) and GMA-grafted (b, c, d) pAAm cryogel. The grafted polymer are visible in the form of rod- and star-shaped particles (b, c and d)

when SEM was used. It should be mentioned, that at present there is no other direct technique suitable for studying the structure of layers of polymer grafted inside the porous hydrogel material in its native hydrated state.

The grafted layer was stained by covalent coupling of fluoresceinamine to epoxy groups and the result was visualized by CLSM, the 3D images were reconstructed representing the structure of the grafted cryogel in the native non-dehydrated state. The CLSM despite somewhat lower resolution than SEM, revealed the same macrostructure of the cryogel as SEM did with thick polymer walls and large pores up to $100 \mu \mathrm{m}$ (Figure 4a). The grafted polymer of GMA was present in the form of particles of different shape (Figure 4b, c, d). CLSM also revealed uneven distribution of grafted GMA in the pores of the cryogel along with the formation of the large rods- and star-shaped particles supporting the mechanism of uneven formation of the initiating centers on the gel surface during the graft polymerization initiated by the initiator deposited on the surface (Figure 4b, c, d). Thus, in order to control grafting from in a rational way it is important not only to control the grafting efficiency i. e. how much polymer is grafted, but it is also vital for the production of the material with desired properties to control how the grafted polymer is distributed along the surface (in this particular case the surface of macropores) used for the grafting.

\section{Conclusions}

GMA was effectively grafted onto the surface of pAAm cryogel using diperiodatocuprate(III) as initiator. The high densities of GMA grafting up to 1.44 mmole of GMA per ml of cryogel or $410 \%$ grafting have been achieved. FTIR, SEM and CLSM confirmed the formation of additional grafted polymer attached to the pore walls of the pAAm cryogel. SEM and CLSM were successfully used for studying of morphology of the gel surface with grafted polymer. CLSM has the advantage over SEM allowing investigations of the distribution of the grafted polymer inside the hydrogel in their native hydrated state. Both microscopic techniques demonstrated uneven distribution of the grafted polymer inside the gel pores as a result of initiating the graft polymerization by insoluble initiator deposited on the pore surface. 


\section{Acknowledgements}

This work was financially supported by Protista International AB (Bjuv, Sweden), The Swedish Foundation for Strategic Research, The Swedish Foundation for International Cooperation in Research and Higher education (STINT, IG2003-2089), The Swedish Research Council (Projects 348-2004-4916 and 621-2005-3604) and The Swedish Institute (Visby Program, project 01211/2004).

\section{References}

[1] Shukla S., Bajpai A. K., Kulkarni R. A.: Preparation, characterization, and water-sorption study of polyvinyl alcohol based hydrogels with grafted hydrophilic and hydrophobic segments. Journal of Applied Polymer Science, 95, 1129-1142 (2005).

[2] Mahdavinia G. R., Zohuriaan-Mehr M. J., Pourjavadi A.: Modified chitosan III, superabsorbency, salt- and $\mathrm{pH}$-sensitivity of smart ampholytic hydrogels from chitosan-g-PAN. Polymers for Advanced Technologies, 15, 173-180 (2004).

[3] Kaneko Y., Nakamura S., Sakai K., Aoyagi T., Kikuchi A., Sakurai Y., Okano T.: Rapid deswelling response of poly( $\mathrm{N}$-isopropylacrylamide) hydrogels by the formation of water release channels using poly(ethylene oxide) graft chains. Macromolecules, 31, 6099-6105 (1998).

[4] Yoshida R., Uchida K., Kaneko Y., Sakai K., Kikuchi A., Sakurai Y., Okano T.: Comb-type grafted hydrogels with rapid deswelling response to temperature changes. Nature, 374, 240-242 (1995).

[5] Kaufmann M.: Unstable proteins: how to subject them to chromatographic separations for purification procedures. Journal of Chromatography B: Biomedical Sciences and Applications, 699, 347-369 (1997).

[6] Müller W.: New ion exchangers for the chromatography of biopolymers. Journal of Chromatography A, 510, 133-140 (1990).

[7] Peppas N. A.: Devices based on intelligent biopolymers for oral protein delivery. International Journal of Pharmaceutics, 277, 11-17 (2004).

[8] Pourjavadi A., Sadeghi M., Hosseinzadeh H.: Modified carrageenan. 5. Preparation, swelling behavior, salt- and pH-sensitivity of partially hydrolyzed crosslinked carrageenan-graft-polymethacrylamide superabsorbent hydrogel. Polymers for Advanced Technologies, 15, 645-653 (2004).

[9] Kumashiro Y., Ooya T., Yui N.: Dextran hydrogels containing poly( $\mathrm{N}$-isopropylacrylamide) as grafts and cross-linkers exhibiting enzymatic regulation in a specific temperature range. Macromolecular Rapid Communications, 25, 867-872 (2004).

[10] Kaneko Y., Sakai K., Kikuchi A., Sakurai Y., Okano T.: Fast swelling/deswelling kinetics of comb-type grafted poly(N-isopropylacrylamide) hydrogels. Macromolecular Symposia, 109, 41-53 (1996).
[11] Kuckling D., Wohlrab S.: Synthesis and characterization of biresponsive graft copolymer gels. Polymer, 43, 1533-1536 (2002).

[12] Lowman A. M., Peppas N. A.: Analysis of the complexation/decomplexation phenomena in graft copolymer Networks. Macromolecules, 30, 4959-65 (1997).

[13] Kim J. H., Lee S. B., Kim S. J., Lee Y. M.: Rapid temperature/pH response of porous alginate-g-poly $(\mathrm{N}$-isopropylacrylamide) hydrogels. Polymer, 43, 75497558 (2002).

[14] Han T. L., Kumar R. N., Rozman H. D., Noor M. A. M.: GMA grafted sago starch as a reactive component in ultra violet radiation curable coatings. Carbohydrate Polymers, 54, 509-516 (2003).

[15] Tjia J. S., Moghe P. V.: Analysis of 3-D microstructure of porous poly(lactide-glycolide) matrixes using confocal microscopy. Journal of Biomedical Materials Research, 43, 291-299 (1998).

[16] Risbud M. V., Bhonde R. R.: Polyamide 6 composite membranes: Properties and in vitro biocompatibility evaluation. Journal of Biomaterials Science. Polymer edition, 12, 125-136 (2001).

[17] Saito H., Nakanishi K., Hirao K., Jinnai H.: Mutual consistency between simulated and measured pressure drops in silica monoliths based on geometrical parameters obtained by three-dimensional laser scanning confocal microscope observations. Journal of Chromatography A, 1119, 95-104 (2006).

[18] Nakayama Y., Matsuda T.: Surface macromolecular architectural designs using photo-graft copolymerization based on photochemistry of benzyl N,Ndiethyldithiocarbamate. Macromolecules, 29, 8622 8630 (1996).

[19] Lozinsky V. I., Galaev I. Y., Plieva F. M., Savina I. N., Jungvid H., Mattiasson B.: Polymeric cryogels as promising materials of biotechnological interest. Trends in Biotechnology, 21, 445-451 (2003).

[20] Jaiswal P. K., Yadava K. L.: Determination of sugars and organic acids with periodato complex of copper(III). Indian Journal of Chemistry, 11, 837-838 (1973).

[21] Savina I. N., Galaev I. Y., Mattiasson B.: Graft polymerization of vinyl monomers inside macroporous polyacrylamide gel, cryogel, in aqueous and aqueousorganic media initiated by diperiodatocuprate(III) complexes. Journal of Polymer Science Part A: Polymer Chemistry, 44, 1952-1963 (2006).

[22] Plieva F. M., Andersson J., Galaev I. Y., Mattiasson B.: Characterization of polyacrylamide based monolithic columns. Journal of Separation Science, 27, 828-836 (2004).

[23] Plieva F. M., Savina I. N., Deraz S., Andersson J., Galaev I. Y., Mattiasson B.: Characterization of supermacroporous monolithic polyacrylamide based matrices designed for chromatography of bioparticles. Journal of Chromatography B: Analytical Technologies in the Biomedical and Life Sciences, 807, 129 437 (2004). 
[24] Plieva F. M., Karlsson M., Aguilar M. R., Gomez D., Mikhalovsky S., Galaev I. Y.: Pore structure in supermacroporous polyacrylamide based cryogels. Soft Matter, 1, 303-309 (2005).

[25] Persson P., Baybak O., Plieva F., Galaev I. Y., Mattiasson B., Nilsson B., Axelsson A.: Characterization of a continuous supermacroporous monolithic matrix for chromatographic separation of large bioparticles. Biotechnology and Bioengineering, 88, 224-236 (2004).

[26] Savina I. N., Galaev I. Y., Mattiasson B.: Graft polymerization of acrylic acid onto macroporous polyacrylamide gel (cryogel) initiated by potassium diperiodatocuprate. Polymer, 46, 9596-9603 (2005).
[27] Savina I. N., Galaev I. Y., Mattiasson B.: Anionexchange supermacroporous monolithic matrices with grafted polymer brushes of N,N-dimethylaminoethylmethacrylate. Journal of Chromatography A, 1092, 199-205 (2005).

[28] Zhang X., Liu W. H., Chen Y. M., Gong A. J., Chen C. F., Xi, F.: Self-condensing vinyl polymerization of acrylamide. Polymer Bulletin, 43, 29-34 (1999).

[29] Roeges N. P. G.: A guide to the complete interpretation of infrared spectra of organic structures. John Wiley and Sons Ltd, Chichester (1994).

[30] Savina I. N., Galaev I. Y., Mattiasson B.: Ionexchange macroporous hydrophilic gel monolith with grafted polymer brushes. Journal of Molecular Recognition, 19, 313-321 (2006). 